\title{
Drying and disaggregation of cotton powder cellulose in the flash drying unit
}

\author{
Bakhtiar Yunusov ${ }^{1}$ \\ ${ }^{1}$ The Tashkent state technical university, after I. Karimov, Tashkent city, Uzbekistan
}

\begin{abstract}
The issues related with the drying and disaggregation of the cotton powder cellulose (CPC) in the flash drying unit are being discussed, as well as the properties of the cotton powder cellulose are being analyzed as the subject of drying and processing treatment.
\end{abstract}

\section{Introduction}

The produced mechanically and chemically the cotton powder cellulose differs with the degree of order, change in the morphological structure and the macroscopic properties [1].

During drying of the powder cellulose with the initial $400 \%$ wetness, the parts of material stick with each other and form big clogs of size 0.0024-0.02 m. The equivalent diameter of its clogs after the drying, before grinding, makes up $d_{e q}=0.008 \mathrm{~m}$. That is why the dried cotton powder cellulose is then milled at the ball grinder for 2 hours, sieved through the sieve No.12 and then packed. The purpose of the work: Inspection of the CPC properties, selection of the type of the equipment for drying, disaggregation and separation of CPC.

\section{The analysis of the properties of the cotton CPC as a subject of the processing and drying.}

The main reason of the lack of the production of the powder cellulose is in the lack of the study of separate technological processes during its production. One of which is the process of drying and grinding, because irrelevant of the method of production of the powder cellulose, its particles are prone to sticking and forming the clogs that must be grinded. Herewith, the process of drying should be revised in the context with other technological treatments of the material and their mutual influence must be considered as well. This will not only intensify and improve the drying process, but recommend the advisable technology of other processing treatments, thus increasing their technical and economical indices as well as the quality of the final product.

Based on the properties of the powder cellulose as the subject of drying, the method of its drying can be proposed observing the sufficiently high degree of activity of the hydrodynamic condition in the drying unit and the time of processing of the material in it.

Different drying equipment is used for drying the disperse polymeric material, that differ in the range of the characteristics constituting the base of their classification [2].

Big quantity of the being dried products together with different methods of heat supply and hydrodynamic conditions, contribute to the variety of the design of the driers thus create the problem of selection of the right drier, type of the drying equipment and the mode of the drying process [3]. The general requirements to the selection of the rational method of drying take to the more profitable technical and economical indices of the operation of the unit, provide reliability of tis operation in producing the high quality product, provide convenient control and maintenance, implementation of the requirements of the safety rules, sanitary and hygiene conditions of labor, exclude the emissions to the atmosphere.

The selection of the rational method of drying shall be supported with the complex of the material characteristics as the subject of drying and the technological peculiarities of production. The main conditions are the technological and economic requirements, as well as the requirements to the quality of the being dried product.

For drying of the disperse materials in the range of the industries, mechanical and low capacity drying units are widely used. Their critical deficiencies are conditioned by the incompliance of the design and operational characteristics used for drying of a particular material.

At the present time, the progressing method of drying in the boiling layer becomes widely used, that allows to sharply intensify the process. However, in many cases, the use of this method largely wears the products, generates static electric charges, as well as create the canals and dead zones. That is why the drying is recommended to be conducted in the flash driers, that are free from the most of the deficiencies 
that the driers with the boiling layers have. The wide introduction of the mentioned driers was prevented by the lack of the correct methods of calculation and rational schemes of installation that allow effective drying of the loose materials. Hence, the proposed methods of calculation provided satisfactory results only in separate cases.

For the first time the prof. Mukhitdinov D.N. mentioned the specifics of the flash driers [4]. The interest to the flash driers in the industry considerably increased after the publication of the works of P.G. Romakova and N.B. Rashkovskoy [8] that demonstrated big possibilities of the mentioned driers.

There are two types of flash driers: without the grate and with it.

The unit without the grate, in the narrow part of it, the gas speed is accepted equal to the stable speed of the pneumatic transportation of big particles, in other words:

$$
\begin{aligned}
& U_{2}=(1,5-:-2,0) U_{B} \\
& U_{B}-\text { speed of the particles. }
\end{aligned}
$$

The cone angle is usually accepted within the range of $30-70^{\circ}$. Based on the data of Elperin I.T. and Ephremtseva V.S., the optimal value of the cone angle shall be calculated based on the formula:

$$
\Theta_{0}=180^{\circ}-2(\varphi+\gamma)
$$

$\varphi$ - the angle of the natural slow of material; $\gamma-$ the angle equal to $30-40^{\circ}$.

The study of the balanced condition of the wet material with the environment facilitates the valuable interest for the evaluation of the moving force of the drying process. Hence the importance is in the analysis of the isotherms of adsorption - desorption. Based on the isotherms of adsorption-desorption define whether the final wetness of the material complies with the even one and hence recommend the conditions of storage of the material, as well as evaluate the porous structure of the material and define the forms of cohesion of the moist with the material.

In order to obtain the isotherms of adsorption and desorption of the materials, we used the vacuum sorption scales. The character of the obtained at $25^{\circ} \mathrm{C}$ isotherms, it is necessary to underline the presence of the node of the sorption hysteresis in the relevant humidity of the air, that justifies the soft structure of the material which is peculiar to the capillary-porous colloid bodies.

Our aim, besides of the analysis of the cohesion forms of the moist in the being studies material, was to determine the energy of the cohesion of water. The energy of cooperation of this material is set within the interval below $3.5 \times 10 \mathrm{~J} / \mathrm{kg}$, which characterizes relevantly weak forms of cohesion of the physicchemical or physics-mechanical types.

To evaluate the porous structure of the material based on the sorption measurements on the Kelvin-
Thomson equity, the differential and integral functions of distribution of the volume of the pours along the radiuses were calculated. On the curves the vital diameter of the pours of $6.0 \mathrm{Nm}$ was defined, in other words the diameter of the thinner pours out of which it is required to remove the moist from the material to obtain the required residual wetness. On the values of the vital diameter of the pours taking into account the adhesion-cohesion properties of the material and based on the forms of cohesion of the moist with the material, the belonging of the material to this or that classification of the disperse materials as the subjects of the drying is defined.

The knowledge of the thermal characteristics is required during processing of the results of the experimental researches of the heat exchange processes during the drying in criterial form, to analyze the mechanism of heat transfer in the wet material, as well as to analyze the forms and types of cohesion of the moist with the material.

In order to determine the TPC (thermal and physical coefficient) of the cotton powder cellulose, we selected the method that is based on the solutions of the nonstationary problems of the heat transfer with the inner source of the heat of constant force.

As seen in the results of the experiments, the heat transfer factor of the being studied material, increases with the increase of the moist content with the tendency to fade at the presence of the moist of the level close to the complete saturation, when the sedimentation of the particles is observed and the difficulties of their stabilizing in the required volume of the liquid occur.

The heat transfer factor of the wet materials increases at the beginning, reaches the maximum at the particular wetness, and then decreases. The explanation of this interdependencies is the following: the dry material has lower thermal and temperature conductance due to the weak thermal contact between its particles. The presence of water improves the thermal contact and considerably eases the transfer of the heat from one particle to the other through thin water film.

In the technological process of production of the cotton powder cellulose, the drying takes an important place. The selection of the drier for this material depends on its properties.

The microscopic research of the structural and mechanical properties of the cotton powder cellulose revealed that its particles represent the spiral fiber, that easily form the aggregates (clogs) that during the drying must be broken into small pieces.

By the thermographic analysis of the hypothermic properties of the cotton powder cellulose it is defined that within the range of the moist content 2.3-:-0.06 $\mathrm{kg} / \mathrm{kg}$ the capillary moist in the pours is removed ( $83 \%$ of the total content of the moist in the material), osmotic moist $(10 \%)$ and the moist of poly-molecular adsorption $(2 \%)$.

The adsorption method of study of the material structure allows to receive the curves of distribution of the volumes of the pours on the radiuses, out of which, based on the Kelvin-Thomson equation, the 
vital diameter of the pours was calculated of $6.0 \mathrm{Nm}$, in other words the smallest diameter of the pours, the removal of moist from which allows reaching the required residual wetness of the material.

Based on the complex analysis of all the properties of the cotton powder cellulose, 3 subgroups were referred to the materials of the II group based on the classification of Mukhitdinov D.N., for which the combined flash drier is recommended.

During the production of the cotton powder cellulose in the industry, the special requirements are set forth to its dispersiveness, hence the process of drying was combined with the grinding of the material. The intensification of the process was reached as a result of renewing and activation of the surface of vaporization during grinding of the cotton powder cellulose. However the time of presence of the particles of the material in the unit is not sufficient to reach the required final wetness of the powder cellulose. That is why for the process of drying and grinding of the cotton powder cellulose in the flash drier, the winded stream of the gas mixtures and neutral additives. The use of the vortex in the supply orifice of the heating service allows to increase the retention capacity and decrease the hydraulic resistance of the layer, which prevents the formation of the canals and dead zones in the chamber.

The peculiar property of such device is that the drying agent at high speed can be injected into the drying chamber, where the vortexed stream is developed. A convection heat exchanging is intensified in it and at intensification of which the special role is assigned to the centrifugal forces that provide not only active mixing, but better grinding. Herewith, the degree of winding of the heat transfer medium plays a vital role for the highly wet materials which stick to the walls of the device. The role of the neutral agents is in "grinding" the particles of the material and in prevention of formation of the clogs, sticking of the particles to the wall of the device, thus providing the movement of the layer. The drying device of this type operates in autonomous mode, in other words, the yet wet and under grinded material is not taken out of the device until it reaches the earlier set parameters of the process.

In this way, the hydrodynamic mode of the flash drier of cone form with the winded stream of the gas mixture and with the neutral additive for the drying and grinding, fully complies with the required property of the material as the subject of the drying.

During the drying of the CPC, the hazardous substances are the dust of the powder cellulose. The powder cellulose is a combustible and explosive substance. The ignition temperature is $210^{\circ} \mathrm{C}$. The lower explosion limit is $50 \mathrm{~g} / \mathrm{m} 3$ in the air. The dust of CPC can damage the health. The base of the hazard: content of $\left(\mathrm{SiO}_{2}\right),(\mathrm{CaO}),\left(\mathrm{Fe}_{2} \mathrm{O}_{3}\right)$ in the dust. The maximum allowable concentration of the dust in the air is $6 \mathrm{mg} / \mathrm{m} 3(\mathrm{CH} 246-63)$. The process name of the product - electrode cellulose. Empirical formula -
$\left(\mathrm{C}_{6} \mathrm{H}_{10} \mathrm{O}_{5}\right)_{\mathrm{n}}$.

Let's calculate the temperature and pressure at the explosion of the cotton powder cellulose. The initial temperature of the mixture is $+20{ }^{\circ} \mathrm{C}$. The initial pressure of the mixture is $760 \mathrm{~mm}$.mrc.clm.Let's write the thermochemical equation of the combustion reaction of the powder cellulose in the air:

$$
\begin{gathered}
\mathrm{C}_{6} \mathrm{H}_{7}(\mathrm{OH})_{3} \mathrm{O}_{2}+6 \cdot 3,76 \mathrm{~N}_{2}= \\
6 \mathrm{CO}_{2}+5 \mathrm{H}_{2} \mathrm{O}+6 \cdot 3,76 \mathrm{~N}_{2} ;
\end{gathered}
$$

The value $\mathrm{Q}_{\text {r }}$, that equates to $4200: 6.173=$ $680.38 \mathrm{kcal} / \mathrm{mole}$, calculated from [5]. The calculation of the explosion temperature is done based on the assumption that the explosion is an adiabatic process that occurs at constant volume. The heat produced during explosion is used only for heating the products of the explosion, based on this assumption, the temperature of the explosion can be calculated by the formula:

$$
Q_{v}=C_{v} \cdot T_{v}
$$

Where: $\left(C_{v}\right)$ - average heating capacity of the products of explosion in the range from $(0)$ to $\mathrm{T}{ }^{0} \mathrm{~K}$ $(v=$ const $) ;\left(T_{v}\right)$ - being searched explosion temperature, $\left({ }^{0} \mathrm{~K}\right) ; Q_{v}$ - explosion heat, $(\mathrm{kcal} / \mathrm{mole})$.

Taking into account, that $\left(T_{v}\right)$ is not known, and $\left(C_{v}\right)$ depends on the temperature, the explosion temperature is calculated based on comparison of the values of the inner energy of the products of the explosion (combustion) with each other from one side and the heat of the explosion (combustion) of the initial substances from the other side. The heat, emitted at the moment of explosion, is contained in the products of the explosion and equates to the increase of the inner energy. Based on this condition, it can be written down:

$$
Q_{v}=Q_{2}+Q_{\text {в.э. }}=\sum m_{i} \Delta E_{\text {m.взр.; }}
$$

Where: $Q_{2}$ - heat of combustion of one mole of the initial substance, taken to the temperature $\left(T_{i}\right)$, [kcal/mole]; $Q_{\text {в.э. }}$ - value of the inner energy of the initial mix at the temperature $\left(T_{i}\right),[\mathrm{kcal} / \mathrm{mole}] ; m_{i}-$ number of moles of $\mathrm{i}$ component in the products of explosion (combustion); $\Delta E_{m .63 p}$. - value of the inner energy of the products of explosion at the temperature $\left[T_{\text {взр. }}{ }^{0} K\right]$.

The inner energy $\left(Q_{\text {в.э. }}\right)$ of the mixture is calculated by the formula:

$$
Q_{\text {в.э. }}=n_{1} C_{v 1} T_{1}+n_{2} C_{v 2} T_{1}+\cdots+n_{i} C_{v i} T_{1} \text {; }
$$

Where: $n_{i}$ - number of moles of each component of the initial mixture; $C_{v i}$ - average heat capacity of 
each component of the mixture at the temperature ( $T$ $\left.{ }^{0} K\right),\left(T_{1}=293{ }^{\circ} K\right) ; \quad C_{\text {v.(yел.) }}=44,152$ [cal $/$ mole.degr]; $C_{v(O 2)}=5,006$ [cal $/$ mole.degr $]$;

$C_{v(v 2)}=4,268[\mathrm{cal} / \mathrm{mole}$.degr]; and:

$$
\begin{gathered}
Q_{\text {в.э. }}=12936,536+8800,548+32838,877= \\
54575,961[\mathrm{cal} .]=54,576[\mathrm{kcal} .]
\end{gathered}
$$

the heat of the explosion of the mixture is calculated by the formula (2)

$$
\begin{aligned}
Q_{v}=Q_{2}+Q_{\text {в.э. }}= & 680,38+54,576=734,956 \\
& {[\text { kcal. }] }
\end{aligned}
$$

The inner energy of the products of the explosion is calculated for the preliminarily set value of the temperature of the explosion. The explosion is the adiabatic process, that is why:

$$
\begin{aligned}
\Delta E= & 6 \int_{0}^{T}\left(C_{v 1}^{\prime}\right) d T+5 \int_{0}^{T}\left(C_{v 2}^{\prime}\right) d T+63,76 \\
& \int_{0}^{T}\left(C_{v 3}^{\prime}\right) d T ;
\end{aligned}
$$

Where: $C^{\prime}{ }_{v 1}, C_{v 2}^{\prime}, C_{v 3}^{\prime}$ - number of products of the explosion (carbon dioxide, water, nitrogen), [mole]

The preliminary heat of the explosion $\left(Q_{в з р}\right)$ is defined on the approximate value of the inner energy of the gases (products of the explosion), obtained by division of $\left(Q_{v}\right)$ to the sum of the products of the explosion;

$$
\begin{aligned}
& Q_{\text {взp. }}=Q_{v} / C_{v i}^{\prime}= 734956 / 25,46=28867[\mathrm{cal} / \mathrm{mole} .]= \\
& 28,867[\mathrm{kcal} / \mathrm{mole}]
\end{aligned}
$$

This value is within the range of $(2000-3400)^{\circ} \mathrm{K}$ [1]. Accepting the $T_{l}=2800{ }^{0} \mathrm{~K}$. Average values $C_{v 1}^{\prime}, \quad C_{v 2}^{\prime}, \quad C_{v 3}^{\prime}$ are calculated based on the pressure of the inner energy [1] to the value of the accepted temperature. For example, for the carbon dioxide gas:

$$
\begin{gathered}
C_{v 1}^{\prime}=Q_{\text {взр }} / T_{1}=28867 / 2800=10,31[\mathrm{cal} \\
\text { /mole.degr }] ; \\
C_{v 2}^{\prime}=8,57[\mathrm{cal} / \text { mole.degr }] \\
C_{v 3}^{\prime}=6,04[\mathrm{cal} / \text { mole.degr }]
\end{gathered}
$$

Hence the average values for water and nitrogen are calculated. By inserting the values $C_{v 1}^{\prime}, \quad C_{v 2}^{\prime}, \quad C_{v 3}^{\prime}$ in the formula [4] we receive:

$$
\begin{gathered}
E_{l}=6 \cdot 10,85 \cdot 2800+5 \cdot 8,57 \cdot 2800+6 \cdot 3,76 \cdot 6,04 \cdot 2800= \\
683788[\mathrm{cal}] .
\end{gathered}
$$

Hence $\quad\left(T=3000^{\circ} \mathrm{K}\right) \quad$ and calculating

$$
\text { ( } E_{2}=740941 \text { cal.) }
$$

By the linear interpolation the explosion temperature is found:

$$
\begin{gathered}
T_{\text {взр. }}=2800+[(734956-683788) /(740941- \\
683788)] \cdot 200=2979{ }^{0} \mathrm{~K} .
\end{gathered}
$$

The pressure generated at the explosion is calculated. The calculation is by the formula:

$$
\begin{aligned}
& P_{\text {взр. }}=\left(P_{0} T_{6 з р} / T_{0}\right) ;(\mathrm{m} / \mathrm{n}) ; \\
& P_{\text {взр. }}=11,54[\mathrm{~atm}] .
\end{aligned}
$$

\section{Conclusion}

The properties of the cotton powder cellulose were researched as the subject of the technological processing and drying. The selection of the type of the drier was made. The calculation method of the device was developed applicable to the CPC, as well as a range of the similar clogging materials. Based on the conducted researches, the pilot device for the drying and disaggregation of CPFC was designed and manufactured. Taking into account the pressure of the explosion, calculated above, the structure of the devices has anti-explosion valve installed in it and in the case of the explosion it discharges the developed pressure.

\section{References}

1) B.Kh. Yunusov* M. B. Aripjanova, N.A. Yunusova. Use of artificial neural network in the process of drying of the cotton powder cellulose. Problems of energy and resource preservation No.12, Tashkent, 2015, p.111.

2) B.Kh. Yunusov, D.N. Mukhitdinov, A.A. Mukolyannts, Sh.B. Yunusov. "Sorption-structural characteristics of the cotton powder cellulose (CPC)", International science conf. "INNOVATION-2011", assembly of science articles, Tash.-2011, 25-27 of October, p.108-109.

3 ) I.N. Pavlov, V.A. Kunichan. Grinding of the micro-crystal cellulose during drying. Chemicstry of the plant raw material. 1999, No.2, p.159-162.

4) B.Kh. Yunusov, D.N. Mukhitdinov. Drying unit with the winded stream of gas mixtures with the neutral additives for drying and grinding of highly wet, clogging materials. TshDTU, "Catalogue of innovation ideas, developments and projects of the professors, teachers and young scientists", Tashkent 2011, p.68.

5) V.S. Saushev, P.P. Sheglov. "Examples and tasks on special chemistry”. Moscow-1971, g.55. 